\title{
Forensic Biomechanics/Neuroscience of Alleged Brain Injury (TBI): A Restaurant Defense Case
}

\section{Patrick Hannon *}

Department of Biological Sciences, Northern Arizona University, Flagstaff, Arizzona, USA

\section{Introduction}

Traumatic brain injury (TBI) can be a very debilitating injury with several mechanisms of injury including linear acceleration, angular acceleration, energy wave propagation and penetration. All TBI mechanisms result in some degree of upper central nervous system strain and/or damage/destruction. Tolerance limits of the brain and brainstem are the challenge for biomechanics' experts, physicians and neuropsychologists. One issue important to the personal injury attorney is the magnitude of the load or force involved in a head strike. The question is whether the level of loading (e.g., angular acceleration, linear acceleration) was sufficient to produce brain injury. Defense and plaintiff's attorneys need to differentiate the client with psychogenic disorders after alleged head trauma from the client with real organic brain injury due to a trauma event. This argument which is outside the academic expertise of the biomechanist/neuroscientist is expressed by Sheftell et al. [1] who report that "In litigious cultures, when there can be active solicitation by attorneys of injury-related cases and patients being coached by attorneys, assessment of malingering may present a challenge even to experienced clinicians and neuropsychologists" [2]. "Malingering as a diagnosis should not be made by exclusion. Observation of tasks not able to be performed, performance on forced choice tests worse than chance, Cluster B personality disorders, prior work history, prior injury claims and excessive endorsement of symptoms may indicate feigning of symptoms" [3].

The plaintiff, a lady in her mid-fifties was a visitor to the city and attending a convention. She and a friend had entered a restaurant for dinner soon after arriving at the hotel. The plaintiff was seated in a long padded bench and her friend was seated in a chair on the other side of the table. The plaintiff indicated that she sat down and had placed her order and a little later she was hit on the back of her head by a full bottle of Champagne and rack that had been placed upon the ledge of her seatback behind and over her head. The total weight of the bottle was 3.24 pounds and the bottle's center of gravity dropped a maximum displacement of 10.5 inches to the back of the plaintiff's head in accord with her approximate sitting height. In discussing the sequence of events, the plaintiff indicated that "when it hit my head, I don't remember it hitting my head right away because the impact was so hard that when it hit my back it knocked all the breath out of me". The bottle and metal display ended up on the bench seat next to the plaintiff. Her friend did not observe this accident event as she indicated that she was looking away at the time that the bottle fell. The plaintiff indicated that much of the evening was not well remembered although she did remember being given a free night's lodging at the hotel by a staff member. The plaintiff did indicate that her head was not bleeding, but indicated there was a huge lump in the spot where she was struck on the head [4] and someone brought her a cloth and some ice for her head. The plaintiff indicated that a significant horizontal force (i.e., a push) must have occurred to the bottle for it to have hit so hard on her upper back to take her breath away. She also thought that the bottle may have been thrown or bumped. She sought medical attention two days after the incident in her home city at a hospital emergency department
(ER). Her head was found to be normocephalic and her neurological exam and a head/brain CT scan were without any positive findings. The plaintiff indicated at ER that she did not have a loss of consciousness and she denied any nausea/vomiting, neck pain or ataxia/syncope. One week later after her ER visit, she was administered a MRA with contrast which found a normal and robust blood flow in key arterial systems within her brain and brainstem. In subsequent months and years, the plaintiff's mental health declined and some symptoms were consistent with traumatic brain injury with an alternative diagnosis of simply aging and some form of somatoform disorder.

We were retained to investigate and analyze the probability of moderate TBI resulting from this accident. Our investigation concluded that the most probable scenario was that one of the larger loop base supports of the bottle rack was moved over the edge of wall top when the plaintiff or someone else bumped up against the side of the seatback wall causing the rack and bottle to fall. The area in back of the seat was occupied by restaurant staff. At our inspection we were able to produce some horizontal vibration motion sufficient to move the large loop base support of the bottle rack over the edge of the seatback top causing the rack and bottle to fall. The wire metal rack was not tightly coupled to the bottle and therefore the rack mass (approximate .1 slug) was not be added in our momentum equation calculation in this subject accident. Our inspection included using an exemplar subject, (same stature as the plaintiff), taking measurements, photographs, and finally video recordings of the falling bottle and rack to the bench seat without the exemplar human subject present.

The 10.5 inch drop of the approximate center of gravity was modeled as a free fall rather than using an inverted pendulum model and the vertical velocity of bottle was found to be approximately $7.5 \mathrm{ft} / \mathrm{second}$ at head contact. The momentum equation was utilized and a worst case scenario assumed that the momentum of the Champagne bottle was brought to zero at contact with the top of the plaintiff's calvarium. In our view, given the testimony of the plaintiff of a glancing blow to the back of her head and in her view a subsequent second significant strike to the right side upper back (close to her right scapula) indicated that only a small fraction of the linear momentum would have been absorbed during the actual head impact. Nevertheless, we assumed this worst case scenario and when realistic time frames were calculated and bracketed to determine the average reaction forces and consequent

${ }^{*}$ Corresponding author: Patrick Hannon, Department of Biological Sciences, Northern Arizona University, Flagstaff, Arizona, USA, Tel: 480-816-0930; E-mail: Hannon@hannonbiomechanics.com

Received April 06, 2018; Accepted April 07, 2018; Published April 14, 2018

Citation: Hannon P (2018) Forensic Biomechanics/Neuroscience of Alleged Brain Injury (TBI): A Restaurant Defense Case. J Forensic Biomed 9: e112. doi: 10.4172/2090-2697.1000e112

Copyright: (C) 2018 Hannon P. This is an open-access article distributed under the terms of the Creative Commons Attribution License, which permits unrestricted use, distribution, and reproduction in any medium, provided the original author and source are credited. 
Citation: Hannon P (2018) Forensic Biomechanics/Neuroscience of Alleged Brain Injury (TBI): A Restaurant Defense Case. J Forensic Biomed 9: e112. doi: 10.4172/2090$2697.1000 \mathrm{e} 112$

Page 2 of 6

potential accelerations applied to the plaintiff's head link, we estimated levels of 1.5 up to 3.0 average g loads. Peak g levels would necessarily have been higher. It was our opinion that a focal point contact of a bottle edge striking the head could have resulted in a "goose-egg" swelling of the posterior SCALP. However, medical records two days after the accident did not provide support for the plaintiff's report of such a swelling or lump.

Finally, the literature describing mild traumatic brain injury (MTBI) was summarized and we opined that even mild TBI or other central nervous system pathology was not the result of this accident. Necessarily, the diagnosis and treatment of this woman was beyond the scope of our expertise and was deferred to the appropriate physician and neuropsychology experts.

\section{References}

1. Sheftell F, Tepper SJ, Lay CL, Bigal ME (2007) Post-traumatic headache: emphasis on chronic types following mild closed head injury. Neurol Sci 28 203-207.

2. Hayes JS, Varney NR, Roberts RJ (1999) Malingering traumatic brain injury: current issues and caveats in assessment and classification. The evaluation and treatment of mild traumatic brain injury. Lawrence Erlbaum Associates, Mahwah, NJ. pp: 249-290.

3. Young WB, Khoury JB, Ramadan NM (2005) Headaches associated with head trauma (post-concussion headache). Chronic Daily Headache of Clinicians, pp 185-197.

4. Solomon S (2005) Chronic post-traumatic neck and head pain. Headache 45 53-67. 